\title{
Research on Sag Online Monitoring System for Power Transmission Wire Based on Tilt Measurement
}

\author{
Xia Xiao*, Yan Xu, Jianghua Zhang, Ken Xu \\ State Key Laboratory of Advanced Electromagnetic Engineering and Technology, \\ College of Electrical and Electronic Engineering, Huazhong University of Science and Technology, Wuhan 430074, China
}

\begin{abstract}
Sag online monitoring system for power transmission wire based on tilt angle measurement was proposed, the mathematical model of catenary for power wire was established. Sag calculation models of power wire in the situation without swinging and swinging were derived. By measuring the power wire axial angle and swing angle with dual-axis tilt sensor, the sag could be accurately calculated with the wire swinging. By testing the sag detection system, the result showed that the sag detection system could meet the engineering requirements of sag measurement.
\end{abstract}

Keywords: Tilt, sag, catenary model, online monitoring

\section{Introduction}

With the continuous development of society, there is more and more power density, industrial electricity consumption increases gradually. In power shortage areas, if relying on local resource generation only, the situation that demand exceeds supply of electricity would appear, which restricts the social development of industry and agriculture, and retards the progress of society. However, after use of the new transmission line method, the cost and environmental issues were becoming increasingly prominent. Improving existing wire conveying capacity would be one of the directions of power grids. Improving the wire conveying capacity, can not only solve the problem of peak or fault short conveying capacity shortage, but also can reduce the new line investment, reduce the number of new lines, and ensure the development of regional economy, which have important social and economic benefits [1-3].

In process of power transmission, wire ampacity is the essence of limiting the transmission capacity of transmission line. In order to prevent overload, the design for the rating capacity of the transmission line, construction is a static, conservative values, which is often based on the most adverse weather conditions. But in fact, such bad weather conditions rarely occurred, so transmission potential of the wire cannot be used efficiently in the vast majority of cases.

As there is a definite relationship between the transmission line sag and transmission power, by measuring the line sag, the transmission power limit could be determined reasonably and accurately, then a more direct judgment, whether transmission power beyond the limit of thermal stability or not, could be achieved. In addition, the sag could vary with the temperature of the line, when the distance of the line sag to the ground vertically or crossing reduced, the safe running of the grid would be affected [2][4].

Therefore, in process of power transmission, by monitoring the transmission wire sag data in real time stability and accurately, energy could be delivered more effectively and reasonably, thus ensuring the security of the power system.

\footnotetext{
* Manuscript received July 15, 2012; revised August 18, 2012.

Corresponding author. Tel.: +86-13627107240; E-mail address: xiaoxiahust@163.com.
} 


\section{Sag Detection Principle Based on the Tilt Measurement}

\subsection{Method of sag measurement}

Sag measurement basic methods include: 1) observation method (commonly using a theodolite or other method); 2) monitoring with GPS; 3) through measuring conductor temperature and stress to determine sag; 4) using projection technique to measure sag; 5) by measuring the tilt of the line to determine sag. A variety of features of sag detection methods are shown in Table 1 [5]-[8].

Table 1. A variety features of sag detection methods

\begin{tabular}{|l|l|}
\hline Sag measurement methods & Features \\
\hline Observation method & $\begin{array}{l}\text { Simple, high degree of accuracy, the need of staff on-site operation is not } \\
\text { conductive to real-time monitoring }\end{array}$ \\
\hline GPS & Accuracy, implement difficult and complex algorithms, expensive \\
\hline Conductor temperature and stress & $\begin{array}{l}\text { Relatively cheap, too many measurement parameters, the algorithm complexity, the } \\
\text { error is larger }\end{array}$ \\
\hline Projection technique & $\begin{array}{l}\text { Simple operation, the need for specialized photographers, image processing } \\
\text { problems, larger error }\end{array}$ \\
\hline Tilt of line & The method is relatively simple, cheap, and the algorithm is simple, less error \\
\hline
\end{tabular}

Through above comparison, the sag detection method based on tilt measurement has more advantages.

Overhead transmission wire hangs between two transmission wire towers. As the distance between the two towers is large, the rigidity of the overhead wire has less effect on the overhanging state. So during engineering calculation of transmission wires, the rigidity of the overhead wire is often ignored, while as a flexible cable. The flexible cable is sufficiently soft, which can only withstand tension but not resist bending. The direction of the tension at any point is consistent with the overhead wires in the axial direction. Theoretically, there are two ways to calculate the sag tension of the overhead wires: catenary and parabola.

In general, in the plain and hilly region, the actual length of the overhead line between two towers is about 5\% larger than the span's. In engineering calculations, often considering that the gravity per unit length of the overhead wire itself is uniform distribution at two suspension points direction, according to this principle, the overhead wire overhanging function is parabolic. While, the gravity of the actual transmission wires are distributed uniformly along their own lines, so during calculation, considering the gravity per unit length of the overhead wire itself being the uniform distribution along their own wires is the most practical way. According to this principle, the overhead wire overhanging function is catenary.

\subsection{Sag measuring catenary modeling}

As shown in Fig.1, A and B are the transmission wire towers, $h_{1}$ and $h_{2}$ are the heights of towers A and $\mathrm{B}$, respectively, $h$ is the height difference, $l$ is the span between the tower A and B , $y_{1}$ is the line segment between the two suspension points, $y_{2}$ is the overhead wire, $\phi$ is the angle between $y_{1}$ and the horizontal plane, $\theta$ is the tilt of the line at the suspension point of the tower $\mathrm{A}$, and $f$ is the sag at any point.

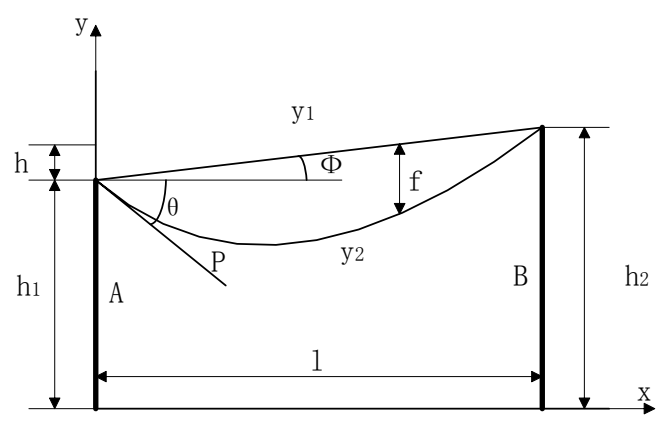

Fig. 1. Transmission line mathematical model without swinging. 
When the wire is not swinging, the wire is located in the plane formed by the two towers.

The wire catenary equation is:

$y=\frac{H}{\omega} \operatorname{ch}\left(\frac{x}{H / \omega}\right)$, where $\operatorname{ch}(x)$ is the hyperbolic function.

The sag $f(x)$ at any point is:

$$
\begin{aligned}
& f(x)=(\tan \phi) x-a \cdot \operatorname{ch}\left(\frac{x-b}{a}\right)+h_{1}-c \\
& a=\frac{l}{\operatorname{arsh}\left(\tan \theta_{A}\right)+\operatorname{arsh}\left(\tan \theta_{B}\right)} \\
& (b / a)=\operatorname{arcsh}(\tan \theta)
\end{aligned}
$$

From the formulas (1), (2) and (3), known the tilt $\alpha$ of the suspension point, the sag at any point could be achieved.

Because of wind or icing, the overhead wires will swing along the connection of the two suspension points as the central axis. At this time, the tilt of the wire will change, the sag derived will differ from the actual sag. The wire catenary model with swinging is shown in Fig. 2.

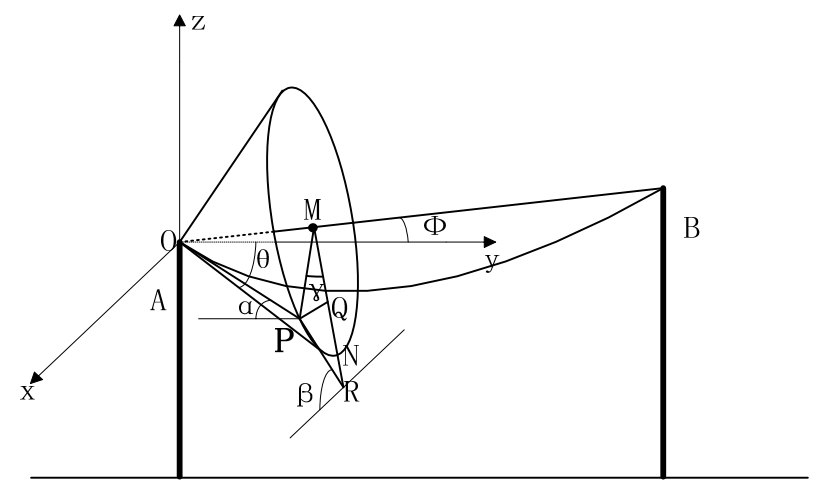

Fig. 2. Wire catenary model with swinging.

In Fig. 2, the overhead wire hangs between the towers A and B, and it's assumed that the location parameters are same as Fig. 1 . When the wire swings, the tracks of the wire tangent is located on the side of the cone which the central axis is the connection between the two suspension points. Let the point where the tangents of wire cuts, and the conical bottom surface be called $\mathrm{P}$, the lowest point of the cone underside be called $\mathrm{N}$, the center of the cone underside be called $\mathrm{M}$, the projection of point $\mathrm{P}$ to the line segment MN be called Q. And the tilt of the wire with swinging is the angle $\alpha$ between the tangent of the wire and the ground plane, the swing angle of the wire is the angle $\beta$ between the tangent $\mathrm{PR}$ on the cone underside and the ground plane. Let the tilt of the wire without swinging be $\theta$, the angle between the connection of the two suspension points and the ground plane be $\phi$.

In the coordinate system $(x, y, z)$, the coordinate of the point $\mathrm{M}$ is $(0,1, \tan \phi)$, the coordinates of the points $\mathrm{P}$ and $\mathrm{R}$ could be achieved respectively:

$$
\begin{aligned}
& \left(\frac{\sin \gamma \tan (\phi+\theta)}{\cos \theta}, 1+\cos \gamma \tan \phi \tan (\phi+\theta), \tan \phi-\cos \gamma \tan (\phi+\theta)\right), \\
& \left(0,1+\frac{\tan \phi \tan (\phi+\theta)}{\cos \gamma}, \tan \phi-\frac{\tan (\phi+\theta)}{\cos \gamma}\right)
\end{aligned}
$$

Then the vector of straight line RP is: 


$$
\begin{aligned}
\overrightarrow{R P}= & \left(x_{P}-x_{R}, y_{P}-y_{R}, z_{P}-z_{R}\right)= \\
& \left(\frac{\sin \gamma \tan (\phi+\theta)}{\cos \phi}, \tan \phi \tan (\phi+\theta)\left(\cos \gamma-\frac{1}{\cos \gamma}\right),-\tan (\phi+\theta)\left(\cos \gamma-\frac{1}{\cos \gamma}\right)\right)
\end{aligned}
$$

And then the sine of the angle between RP and the ground plane is:

$$
\sin \beta=\frac{\overrightarrow{R P} \cdot \vec{n}}{|R P| \cdot|n|}=\sin \gamma \cdot \cos \phi, \quad \sin \gamma=\frac{\sin \beta}{\cos \phi}
$$

The vector of OP is:

$$
\begin{aligned}
\overrightarrow{\mathrm{OP}}= & \left(x_{P}-x_{O}, y_{P}-y_{O}, z_{P}-z_{O}\right)= \\
& \left(\frac{\sin \gamma \tan (\phi+\theta)}{\cos \phi}, 1+\cos \gamma \tan \phi \tan (\phi+\theta), \tan \phi-\tan (\phi+\theta) \cos \gamma\right)
\end{aligned}
$$

Then

$$
\sin \alpha=\frac{\overrightarrow{O P} \cdot \vec{n}}{|\overrightarrow{O P}| \cdot|\vec{n}|}=(\cos \gamma \tan (\phi+\theta)-\tan \phi) \cos (\phi+\theta) \cos \phi, \cos \gamma=\frac{\sin \alpha+\sin \phi \cos (\phi+\theta)}{\cos \phi \sin (\phi+\theta)}
$$

From the formulas (4) and (5), then

$$
\cos (\theta+\phi)=\frac{-\sin \alpha \cdot \sin \phi+\sqrt{(\sin \alpha \cdot \sin \phi)^{2}-\cos ^{2} \beta\left(\sin ^{2} \alpha+\sin ^{2} \beta-\cos ^{2} \phi\right)}}{\cos ^{2} \beta}
$$

In formula (6), $\theta, \beta, \phi$ are respectively the actual tilt, the swinging angle, the angle between the connection of the two suspension points and the ground plane with the wire swinging. Therefore, if the actual tilt and the swing angle of the wire are measured by sensor, and the angle between the connection of the two suspension points and the ground plane could be acquired from engineering parameters, the tilt of the wire without swinging could be deduced conveniently. Then the sag of the wire could be achieved according to the model of the wire without swinging.

\section{Sag Measurement System}

Combining wire construction parameters, the sag online monitoring system based on the angle measuring principle could obtain the sag value at the lowest point of the wire and the maximum sag, and the dual-axis tilt sensor is used to measure the tilt of the wire to the ground and swing angle simultaneously. The sag online monitoring system diagram as shown in Fig. 3, tilt sensor would be mounted coaxially with the overhead wires, obliquity sensor output data is transmitted to the monitoring center by the wireless data transmission system.

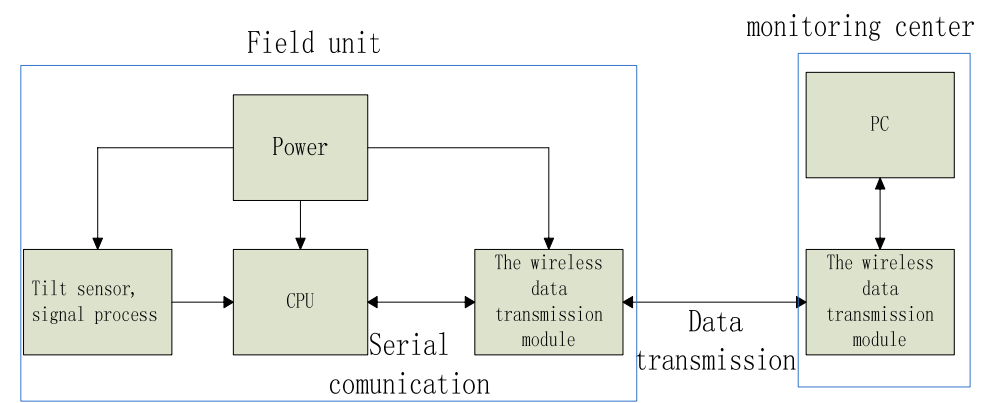

Fig. 3. Sag online monitoring system diagram. 
The selected dual-axis tilt sensor is a acceleration measurement principle sensor, whose measurement range is $\pm 60^{\circ}$, which owns both analogue and digital output, whose analogy output resolution is $0.0025^{\circ}$, whose digital output resolution of $0.035^{\circ}$. And the sensor has a built-in temperature sensor, which could compensate the sensor's temperature dependency by the environment temperature. The anti-seismic strength is $20000 \mathrm{~g}$, the width of $-3 \mathrm{~dB}$ is $8 \sim 28 \mathrm{~Hz}$.

The output data of the sensor through a signal processing circuit is transmitted to the monitoring center through the GPRS wireless network. At the monitoring center, data are collected, arranged, analyzed and stored. And at the same time, when the sag data is found abnormal, the command for collecting sag data intensively will be sent to the field unit, and then a warming will be send out.

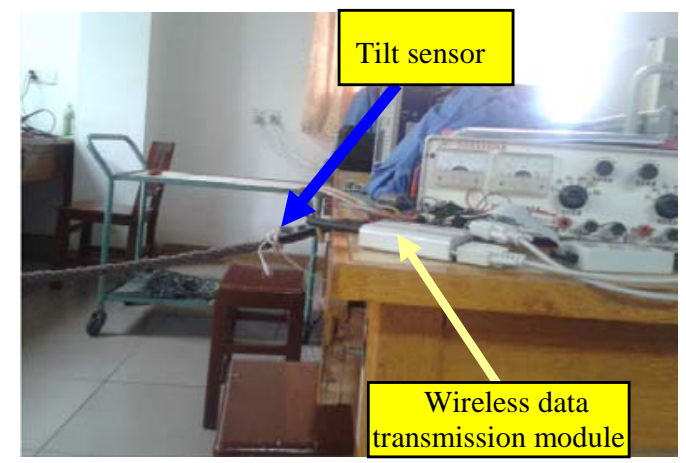

Fig. 4. Sag test system in lab.

\section{Test Result}

The limitations of the experimental conditions, the test program uses a thick rope instead of overhead wires, thick rope is hanging in the space of $3 \mathrm{~m}$ width, and there are height differences between the two suspension points, the tilt sensor with signal processing circuit is bound with the rope coaxially, as shown in Fig. 4. Sag measurement results are transmitted to the monitoring center by wireless remote data transmission module. Changing the sag of the thick rope, the sag is measured at the thick rope actually sag value of $0.069 \mathrm{~m}, 0.124 \mathrm{~m}, 0.096 \mathrm{~m}$ respectively, the test results form the monitoring center are shown in Table 2.

The test results in Table 2 show that the relative sad error is $-0.32 \%$, which meets the sag monitoring requirements engineering.

Table 2. Sag test data

\begin{tabular}{|c|c|c|c|c|}
\hline $\begin{array}{c}\text { Actual sag } \\
\text { value } f_{a}(\mathrm{~m})\end{array}$ & $\begin{array}{c}\text { Maximum sag } \\
\text { value } f_{\max }(\mathrm{m})\end{array}$ & $\begin{array}{c}\text { Sag value at the } \\
\text { lowest point } f_{\text {low }}(\mathrm{m})\end{array}$ & $\begin{array}{c}\text { Relative error of the maximum sag } \\
e_{\max }=\left(f_{\max }-f_{a}\right) / f_{a}^{*} 100 \%\end{array}$ & $\begin{array}{c}\text { Relative error of the sag at the } \\
\text { lowest point } \\
e_{\text {low }}=\left(f_{\text {low }}-f_{a}\right) / f_{a} * 100 \%\end{array}$ \\
\hline 0.069 & 0.0690 & 0.0690 & 0.0000 & 0.0000 \\
\hline 0.069 & 0.0688 & 0.0688 & -0.2899 & -0.2899 \\
\hline 0.069 & 0.0690 & 0.0689 & 0.0000 & -0.1449 \\
\hline 0.095 & 0.0948 & 0.0948 & -0.2105 & -0.2105 \\
\hline 0.095 & 0.0948 & 0.0949 & -0.2105 & -0.1053 \\
\hline 0.095 & 0.0948 & 0.0948 & -0.2105 & -0.2105 \\
\hline 0.124 & 0.1236 & 0.1237 & -0.3226 & -0.2419 \\
\hline 0.124 & 0.1237 & 0.1238 & -0.2419 & -0.1613 \\
\hline 0.124 & 0.1236 & 0.1237 & -0.3226 & -0.2419 \\
\hline
\end{tabular}

\section{Summary}

Overhead transmission wires sag has the corresponding relationship with the transmission capacity, real-time monitoring of the sag would contribute to wire dynamic capacity and improve the system security. The sag detection technique, based on the tilt measurement principle, using mathematical model 
of the catenary has higher accuracy. The test shows that the sag detection system could meet the project sag measurement requirements.

\section{References}

[1] Malhara S, Vittal V. Mechanical state estimation of overhead transmission lines using tilt sensors. IEEE Transactions on Power Systems, 2010; 25(3):1282-1230.

[2] Seppa TO. A practical approach for increasing the thermal capabilities of transmission lines. Power Delivery, 1993; 8(3): 1536-1550.

[3] Huang XB, Sun QD, Han XY. An on-line monitoring system of transmission line conductor de-icing. Industrial Electronics and Applications, 2008; 5(2):1522-1527.

[4] Douglass DA, Edris AA. Real-time monitoring and dynamic thermal rating of power transmission circuits. Power Delivery, 1993; 11(3):1407-1418.

[5] Vijaya Saradhi MV, Nagaraju S. Development of a low-cost ZIGBEE and GSM SMS-based conductor temperature and sag monitoring system. International Journal of Engineering Science and Technology, 2005; 11(2):1427-1451.

[6] Malhara S, Vittal V. Mechanical state estimation of overhead transmission lines using tilt sensors. Power Systems, 2010; 25(3):1282-1290.

[7] Liu YF, Zhang LG, Wang J. Computation of standard sag of overhead lines for power grid based on mathematical model of iterative technique. Computer Engineering and Technology, 2010; 5(2):445-448.

[8] Motlis Y. A new method for real-time monitoring of high-voltage transmission-line conductor sag. Power Systems, 2007; 12(3):1432-1440. 